\title{
Autopsy Domain
}

National Cancer Institute

\section{Source}

National Cancer Institute. Autopsy Domain. NCI Thesaurus. Code C49565.

A subject domain utilized for the submission of information encompassing and representing data, vocabulary or records related to autopsy. 